\title{
Monotone Type Discrete Analogue for the Mixed Boundary Value Problem
}

\author{
By V. Thuraisamy*
}

\begin{abstract}
This paper is concerned with the formulation of finite-difference analogues of mixed boundary value problems for Poisson's equation. A discrete approximation to the normal derivative is devised such that the matrix of the resulting system is of monotone type. This enables us to prove that the rate of convergence is $O\left(h^{2}\right)$, where $h$ is the mesh constant.
\end{abstract}

1. Introduction. In this paper we are concerned with the following problem. $R$ is a bounded domain, in the plane $E_{2}$, with its boundary $\partial R$ suitably smooth. $\partial R$ is made up of $\partial R_{1}$ and $\partial R_{2}$. For $P=P(x, y) \in \bar{R}=R \cup \partial R$ we seek a function $u(P)$ which satisfies the equations

$$
\begin{aligned}
-\Delta u(P) & =f(P), & & P \in R, \\
\partial u(P) / \partial n+\alpha(P) u(P) & =g_{1}(P), & & P \in \partial R_{1}, \\
u(P) & =g_{2}(P), & & P \in \partial R_{2},
\end{aligned}
$$

where $\Delta=\partial^{2} / \partial x^{2}+\partial^{2} / \partial y^{2}, \partial / \partial n$ is the outward normal derivative and $f, g_{1}, g_{2}$ are appropriately smooth given functions. $\alpha(x) \geqq 0$ is piecewise differentiable on $\partial R_{1}$.

This problem (sometimes with $f \equiv 0$ ) has been discussed in [3] where questions such as regularity assumptions, isolated singularities and exterior problems are studied for $R$ in space $E_{n}, n \geqq 2$. The main work here is centered around the development of a new second-order approximation to the normal derivative and establishing that the resulting matrix of the discrete system possesses a nonnegative inverse. This matrix is not of positive type [1].

Higher-order schemes for this problem were studied by Bramble and Hubbard [2] (whose notations are followed here) where they had to restrict the mesh size $h$ severely to guarantee the positivity of the matrix. Also, at each boundary mesh point, several systems of simultaneous equations had to be solved to select three of the nearby points (within 10h) in order to write the corresponding equation. In the method given here, points can be chosen a priori. The restriction imposed in [2] that on $\partial R_{1}$ if $\alpha(P) \neq 0$, it should be bounded away from zero, is also removed. When the solution of (1.1) is sufficiently smooth, the convergence is shown to be $O\left(h^{2}\right)$.

2. Discrete Analogue. With uniform mesh, the set $R_{h}$ of grid points in $R$ is divided between $R_{h}{ }^{\prime}$ and $R_{h}{ }^{*}$ where the latter constitutes the points which are at

Received January 16, 1968, revised July 1, 1968.

* This research was supported in part by the U.S. Atomic Energy Commission under Contract AEC-AT-(40-1) 3443 and by the National Science Foundation under Grant NSF-GP-6631 and was part of the author's Ph.D. thesis at the University of Maryland under the direction of Professor B. E. Hubbard. 
distance less than $h$ from $\partial R_{1}$ measured along the mesh lines. We then write $\bar{R}_{h}=\partial R_{h} \cup R_{h}$, where $\partial R_{h}$ is the set common to $\partial R$ and the grid lines.

In describing the construction of the boundary operator, we shall, for definiteness, consistently refer to Fig. 1(a). The boundary is assumed to have a continuously turning tangent. 0 is the point on $\partial R_{1, h}$ where we wish to approximate the normal derivative. $0 N$ is the normal at $0.0 L, 0 M$ are the two lines making angles of $45^{\circ}$ on either side of $0 N$. Let $0 L$ make an angle $(\pi / 2-\theta)$ with the positive $x$-axis. We pick a point $A$ on $0 L$ which also lies on a mesh line, and a point $B$ on $0 M$ such that the length $\overline{0} \bar{A}$ equals the length $\overline{0} \bar{B}$. In general, $B$ would not be on a mesh line. We let $C$ and $D$ be the nearest points to $B$ that are on $0 M$ and also on mesh lines. We now pick nine points in $\bar{R}_{h}$ which are all within $O(h)$ of 0 and label them as in the figure, where, several of them may be actually on $\partial R$. We also note that although for the sake of clarity we shall continue to talk of these nine points as distinct, it can happen that two or more of these points are in fact the same mesh points [e.g., see Figs. 1(b) and 1(c)].

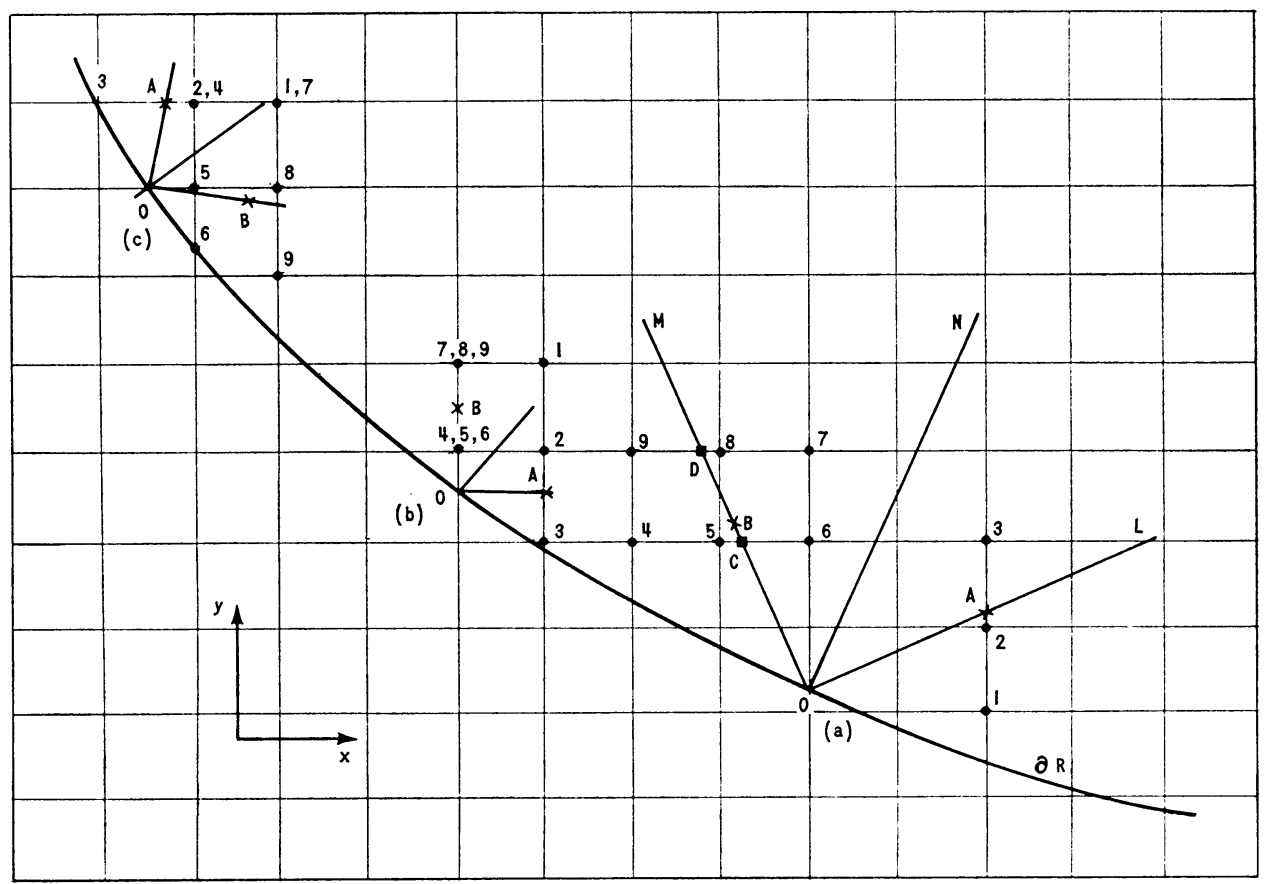

FIGURE 1

We shall write (with $\overline{1} \overline{2}$, e.g., being distance between points 1 and 2)

$$
\begin{aligned}
\overline{1} \overline{2} & =\xi_{1} h, \quad \overline{2} \bar{A}=\lambda_{1} h, \quad \bar{A} \overline{3}=\mu_{1} h, \\
h \operatorname{cosec} \theta & =h_{2}, \quad \overline{0} \bar{C}=\xi_{2} h_{2}, \quad \bar{C} \bar{B}=\lambda_{2} h_{2}, \quad \bar{B} \bar{D}=\mu_{2} h_{2}, \\
\overline{4} \overline{5}=\xi_{3}, & \overline{5} \bar{C}=\lambda_{3}, \quad \bar{C} \overline{6}=\mu_{3}, \\
\overline{7} \overline{8}=\xi_{4}, & \overline{8} \bar{D}=\lambda_{4}, \quad \bar{D} \overline{9}=\mu_{4},
\end{aligned}
$$

where $\lambda_{i}+\mu_{i} \equiv \alpha_{i}, i=1,2,3,4$ and the quantities $\alpha_{i}, \xi_{i}$ are such that $0 \leqq \alpha_{i}$, 
$\xi_{i} \leqq 1$ except that $\alpha_{2} \equiv 1$ and $\xi_{2}(>0)$ may exceed unity. If we define

$$
\begin{aligned}
a_{i} & =-\left(\xi_{i}+\lambda_{i}\right)\left(\xi_{i}+\alpha_{i}\right) /\left(\lambda_{i} \alpha_{i}\right), \\
b_{i} & =-\left(\xi_{i}+\lambda_{i}\right) \xi_{i} /\left(\mu_{i} \alpha_{i}\right), \quad i=1,2,3,4, \\
c_{i} & =1+a_{i}+b_{i},
\end{aligned}
$$

then for $u \in C^{3}(\bar{R})$ we have

$$
\begin{aligned}
& u(A)=\left[u(1)+a_{1} u(2)+b_{1} u(3)\right] / c_{1}+O\left(h^{3}\right), \\
& u(B)=\left[u(0)+a_{2} u(C)+b_{2} u(D)\right] / c_{2}+O\left(h^{3}\right), \\
& u(C)=\left[u(4)+a_{3} u(5)+b_{3} u(6)\right] / c_{3}+O\left(h^{3}\right), \\
& u(D)=\left[u(7)+a_{4} u(8)+b_{4} u(9)\right] / c_{4}+O\left(h^{3}\right) .
\end{aligned}
$$

Using (2.8) and (2.9) in (2.7) gives

$$
\begin{aligned}
u(B)=\left\{u(0)+a_{2}(u(4)\right. & \left.+a_{3} u(5)+b_{3} u(6)\right) / c_{3} \\
& \left.+b_{2}\left(u(7)+a_{4} u(8)+b_{4} u(9)\right) / c_{4}\right\} / c_{2}+O\left(h^{3}\right) .
\end{aligned}
$$

Letting $p$ be the perpendicular distance of 0 from the line joining $A$ and $B$, we easily verify that

$$
\frac{u(0)-(u(A)+u(B)) / 2}{p}=\frac{\partial u(0)}{\partial n}-\frac{p}{2} \Delta u(0)+O\left(p^{2}\right) .
$$

For the convenience of possible users we give (2.11) in its final form below in which we have written $\xi_{i}+\lambda_{i}=\omega_{i}$ and $\xi_{i}+\alpha_{i}=\tau_{i}, i=1,2,3,4$.

$$
\begin{aligned}
u(0)\left(1+\frac{\lambda_{2} \mu_{2}}{2 \xi_{2} \tau_{2}}\right) & +\frac{\lambda_{1} \mu_{1}}{2 \xi_{1} \tau_{1}} u(1)-\frac{\omega_{1} \mu_{1}}{2 \alpha_{1} \xi_{1}} u(2)-\frac{\omega_{1} \lambda_{1}}{2 \alpha_{1} \tau_{1}} u(3) \\
& +\frac{\omega_{2} \mu_{2}}{2 \xi_{2}} \frac{\lambda_{3} \mu_{3}}{\xi_{3} \tau_{3}} u(4)-\frac{\omega_{2} \mu_{2}}{2 \xi_{2}} \frac{\omega_{3} \mu_{3}}{\xi_{3} \alpha_{3}} u(5)-\frac{\omega_{2} \mu_{2}}{2 \xi_{2}} \frac{\omega_{3} \lambda_{3}}{\alpha_{3} \tau_{3}} u(6) \\
& +\frac{\omega_{2} \lambda_{2}}{2 \tau_{2}} \frac{\lambda_{4} \mu_{4}}{\xi_{4} \tau_{4}} u(7)-\frac{\omega_{2} \lambda_{2}}{2 \tau_{2}} \frac{\omega_{4} \lambda_{4}}{\xi_{4} \alpha_{4}} u(8)-\frac{\omega_{2} \lambda_{2}}{2 \tau_{2}} \frac{\omega_{4} \lambda_{4}}{\alpha_{4} \tau_{4}} u(9) \\
& =p \frac{\partial u}{\partial n}(0)-\frac{p^{2}}{2} \Delta u(0)+O\left(h^{3}\right) .
\end{aligned}
$$

We now pose the discrete problem. For the Laplacian we take the well-known five point operator $\Delta_{h}$ in $R_{h}{ }^{\prime}$ and its modified five point form for points of $R_{h}{ }^{*}$ (the $\Delta_{h}{ }^{(1)}$ of [3]). This is known to have local error $O\left(h^{2}\right)$ in $R_{h}{ }^{\prime}$ and $O(h)$ in $R_{h}{ }^{*}$. For $(x, y) \in \partial R_{1, h}$ we define

$$
B_{h} u(x, y) \equiv \frac{1}{p(0)} \sum_{i=0}^{9} d_{i} u(i) \equiv \sum_{i=0}^{9} c_{i} u(i)
$$

where $u(0) \equiv u(x, y)$ and $p(0)$ is the value of $p$ associated with $(x, y)$.

$$
d_{0}=1+\frac{\lambda_{2} \mu_{2}}{2 \xi_{2} \tau_{2}}+\alpha(0) p(0)
$$

$d_{i}, i=1, \cdots, 9$, are the coefficients of $u(i)$ in (2.12). The difference analogue of problem (1.1) now reads 


$$
\begin{aligned}
-\Delta_{h} u(P) & =f(P) \quad \text { in } R_{h}, \\
B_{h} u(P) & =g_{1}(P)-p f(P) / 2 \quad \text { on } \partial R_{1, h}, \\
u(P) & =g_{2}(P) \quad \text { on } \partial R_{2, h} .
\end{aligned}
$$

If $A$ is the matrix of the system (2.15) then there is a diagonal matrix $D$ such that $\bar{A}=D A$ has unit diagonal. Typical patterns of $\bar{A}$ are shown in Fig. 2.

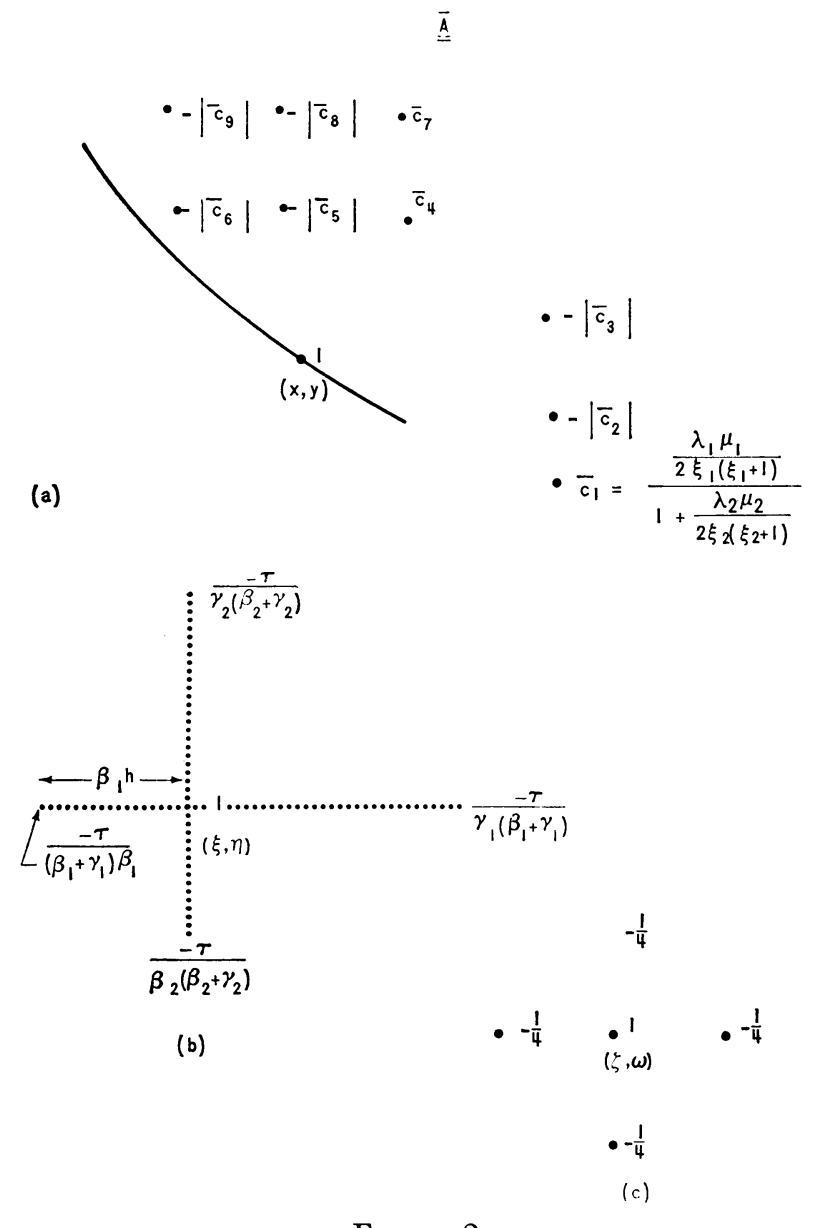

Figure 2

3. Monotonicity of the Matrix $A$. We now state known sufficient conditions for a matrix to be monotone type [1].

Theorem 1 (Bramble-Hubbard). Let $B$ be a square matrix having unit diagonal and such that

$$
\sum_{j} b_{i j} \geqq 0,
$$

with strict inequality holding for $j \in J(B) \neq \varnothing$. Assume that it is possible to write $B$ as the matrix sum $\mathrm{I}-\mathrm{H}_{1}-\mathrm{H}_{2}$, where $\mathrm{I}$ is the unit matrix, such that 
(a) $\left(H_{1}\right)_{\alpha \alpha}=0$ (i.e., diagonal entries are zero),

(b) $I-H_{1}$ is of positive type,

(c) $\left(I-H_{1}\right)^{-1} H_{2} \geqq 0$,

(d) for each $k \notin J(B)$ there exists a connection in $H_{1}$ from $k$ to $J(B)$; i.e., $\exists$ a sequence of nonzero elements $h_{k_{1} 1}, \cdots, h_{k_{r} j}$ where $j \in J(B)$.

Then $B$ is monotone.

With the help of this theorem we shall now establish that our matrix $A$ is monotone.

Theorem 2. A is a monotone matrix provided that the point $A$ in Fig. 1 is chosen such that the $\mu_{i}$ and $\xi_{i}, i=1,2,3,4$, meet conditions (3.3), (3.5)-(3.8) together with occasional remarks in the neighborhoods of these conditions as described in the proof.

Proof. Writing $\bar{A} \equiv I-H_{1}-H_{2}$, it will be convenient to refer to diagrams giving typical patterns of $H_{1}$ and $H_{2}$. We have done this for $H_{2}$ in Fig. 3. For $(x, y)$ $\in \partial R_{1, h}$ the possible nonzero entries of $H_{1}$ would come from the 'connections' 2,5 and 8 , their values being simply the corresponding coefficients $\bar{c}_{i}$, where $\bar{c}_{i}$ are the results of normalizing $c_{i}$ of (2.13) in going from $A$ to $\bar{A}$.

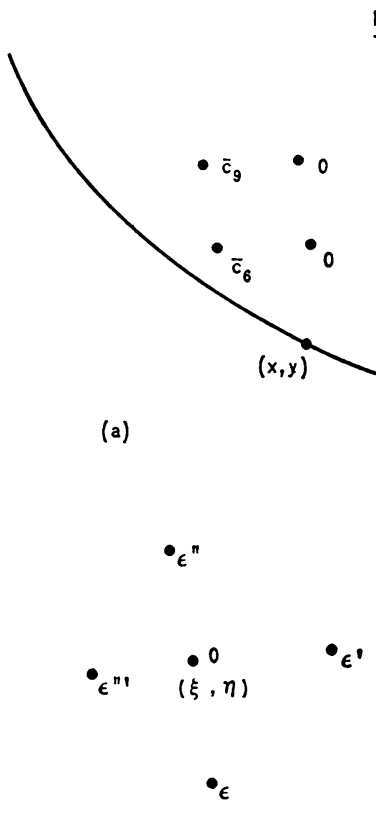

$\underline{H_{2}}$

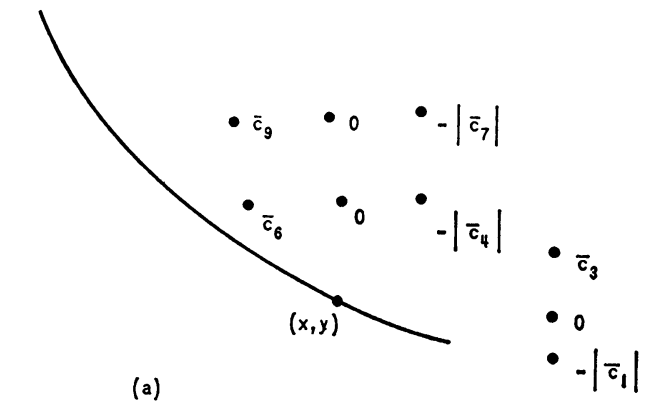

(b)
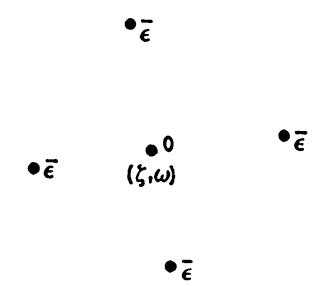

(c)

Figure 3

For $(\xi, \eta) \in R_{h}{ }^{*}$, we go to Fig. $2 \mathrm{~b}$. By the definition of $\Delta_{h}, \beta_{1}, \beta_{2}, \gamma_{1}, \gamma_{2}$ are in $(0,1]$ with at least one of them exactly unity. $\tau$ is the quantity $\beta_{1} \gamma_{1} \beta_{2} \gamma_{2} /\left(\beta_{1} \gamma_{1}+\beta_{2} \gamma_{2}\right)$. 
Thus the $\epsilon$ 's in Fig. 3b can be chosen small enough and positive to leave each of the off-diagonal entries positive (nonnegative would suffice) for $H_{1}$.

For $(\zeta, \omega) \in R_{h}{ }^{\prime}$ we subtract off a quantity $\bar{\epsilon}$ from the absolute value of the off-diagonal entries of $\bar{A}$ such that the remainders are still positive. Once $H_{1}$ is chosen in this manner, the identity $\bar{A}=I-H_{1}-H_{2}$ automatically determines $H_{2}$.

To continue with the proof we first note that conditions (a) and (d) of Theorem 1 hold for $\bar{A}$ as seen from the construction of $H_{1}$. The validity of (b) may be verified from the definition of a positive type matrix, but requires the assumption

$$
\xi_{i} \geqq \mu_{i}, \quad i=1,2,3,4 .
$$

The nontrivial part of the proof is to verify that $\left(I-H_{1}\right)^{-1} H_{2} \geqq 0$. We expand this product as

$$
\left(I-H_{1}\right)^{-1} H_{2} \equiv H_{2}+H_{1} H_{2}+H_{1}^{2} H_{2}+\cdots,
$$

the validity of this expansion being seen easily from the Gershgorin's inclusion theorem (or see [4, p. 46]).
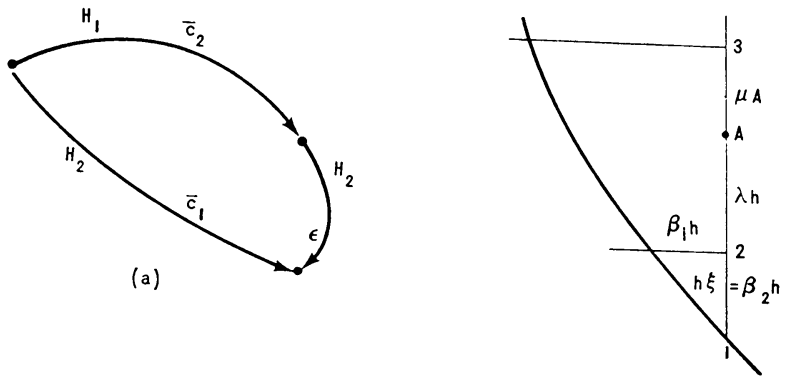

(b)

FigURE 4

The only negative terms of $H_{2}$ occur in 'connections' corresponding to points $(x, y) \in \partial R_{1, h}$, the magnitudes of these terms depending on several factors which we now examine briefly. For the sake of brevity we assume (and this is of no significance in practice) that

$$
\text { either } \xi_{i} \text { or } \alpha_{i} \text { is unity for } i=1 \text { and } i=3 \text {. }
$$

For $-\left|\bar{c}_{1}\right|$. Our first aim is to show that $A$ may be chosen such that the negative term $\bar{c}_{1}$ in $H_{2}$ is cancelled out by the positive term coming out of $H_{1} H_{2}$. E.g., let $\alpha_{1}=1$, ' 1 ' $\in \partial R_{h}$. Then from (2.12), we need an $\epsilon$ simultaneously satisfying (Fig. 4)

$$
\frac{\lambda_{1}+\xi_{1}}{2 \xi_{1}} \mu_{1} \epsilon>\frac{\lambda_{1} \mu_{1}}{2 \xi_{1}\left(\xi_{1}+1\right)}
$$

where $\xi_{1}$ is the $\beta_{2}$ of Fig. 2 and (Figs. 1(b), 3(b))

$$
\epsilon<\gamma_{1} \beta_{1} /\left(\gamma_{1} \beta_{1}+\beta_{2}\right)\left(\beta_{2}+1\right) .
$$

Such an $\epsilon$ exists iff $\lambda_{1}<\beta_{1} \gamma_{1}$. In general we can summarize the situations in all possible cases as follows. Without loss of generality we assume $A$ to be on a line in the $y$-direction and $C, D$ on lines in the $x$-direction. Then an $\epsilon$ (or an appropriately 
adorned $\epsilon$ ) can be found within the permitted range iff

$$
\lambda_{1}<\beta_{1} \gamma_{1} / \alpha_{1} \text {. }
$$

For $-\left|\bar{c}_{4}\right|$. Let us assume that $\gamma_{1}=\gamma_{2}=1$, the contrary happening only with an exceptionally reckless choice of $A$. Then we find that

$$
\lambda_{3} \omega<\beta_{2}
$$

is sufficient, $\omega$ being unity or $\beta_{1}$ according as $\xi_{3}=\beta_{1}$ or $\alpha_{3}=\beta_{1}$.

For $-\left|\bar{c}_{7}\right|$. The situation here, obviously, is analogous to that of $-\left|\bar{c}_{4}\right|$. Since $\xi_{2}+1>1$, ' 8 ' will almost always be in $R_{h}$ '. The possible cases are

(i) $\alpha_{4} \leqq 1, \xi_{4}=1$,

(ii) $\alpha_{4}=1, \xi_{4}<1$.

There is no constraint on the choice of $A$ in case (i). In case (ii), which can happen only when $\xi_{2}<1$, we must require that

$$
\beta_{2}>\lambda_{4} \text {. }
$$

We must bear in mind that in the above discussion we have constantly referred to Figs. 2 and 3 and the numbers $\beta_{1}, \beta_{2}, \gamma_{1}, \gamma_{2}$ correspond respectively to ' 2 ', ' 5 ' or ' 8 ' according to whether we are considering $\bar{c}_{1}, \bar{c}_{4}$ or $\bar{c}_{7}$. The above analysis remains entirely valid for the case of one or more of the nine points coinciding, since the terms in $H_{1} H_{2}$ required to dominate the term in $H_{2}$ remain uncompromised by superposition. Also we note that often an interior point may be involved in more than one equation of type (2.13), but the $\epsilon$ 's can still be found without any further restrictions on the choice of $A$.

To complete the proof: We already have $H_{2}+H_{1} H_{2}$ nonnegative. Writing (3.4) as

$$
\left(I-H_{1}\right)^{-1} H_{2}=\sum_{n=0}^{\infty}{H_{1}}^{2 n}\left(H_{2}+H_{1} H_{2}\right)
$$

and using the fact that $H_{1}{ }^{2 n}$ is positive for all $n$, we see that $\bar{A}$ meets also condition (c) of Theorem 1 . Since $D$ is a diagonal matrix with positive diagonal elements, $A$ must also be of monotone type.

4. Error Estimates. Given the monotonicity of $A$, we may proceed to define a Robin's function, write down a representation formula and establish bounds for discrete sums of the Robin's function over $R_{h}{ }^{\prime}$, etc., exactly as in [3]. Therefore we merely state the conclusion as

THEOREM 3. Let $u \in C^{4}(\bar{R})$ be the solution of problem $(1.1)$. Let $u(P, h)$ be the solution of system (2.15). Then the error $e(P, h) \equiv u(P)-u(P, h)$ satisfies

$$
|e(, h)| \bar{R}_{h} \leqq K h^{2} .
$$

Remark. It is important to note that if $r_{0}$ is the infimum of the positive radii of curvature (i.e., when circles of curvature $\subset \bar{R}$ ) of $\partial R$, then for any ' 0 ' $\in \partial R_{1, h}$ it is always possible to choose $A$ such that ' 2 ', ' 5 ' and ' 8 ' would be in $R_{h}^{\prime}$ ' provided $5 h<r_{0}$. With such a choice of $A$ all restrictions encountered in the proof of the monotonicity theorem are removed. This can be quite easily seen by working with a circle of radius $5 h$. 
It will be easy to doubt the viability of a method that involves nine points to approximate the boundary operator. In fact, however, because of the simple pattern in the coefficients of (2.17), they are determined quickly and easily once the point $A$ is picked wisely.

The only numerical experiment carried out for our scheme was a simple-minded one where we took for $R$ the unit disc with center at $(c, 0)$ and used $\frac{1}{2} \log \left(x^{2}+y^{2}\right)$ as $u(x, y) . \partial R_{1}$ was a semicircular arc and $\alpha(x)$ was unity there. A rather coarse mesh was taken with $h=0.2$. This together with the symmetry of the function about a diameter resulted in a matrix of order 54 (effective order 46, excluding points on $\partial R_{2}$ ). The system of linear equations was solved by the point Gauss-Seidel iterative method [4] (about 70 secs. on IBM 7094) and results compared with the exact values. For $c \geqq 3$ there were only two points where the relative error exceeded $2 \%$ (max value $2.4 \%$ ) and at most points this was down to less than one percent. As $c$ approached unity, the expected deterioration was noticed.

Bellcomm, Inc.

Washington, D. C. 20024

1. J. H. Bramble \& B. E. HubB.ARD, "New monotone type approximations for elliptic problems," Math. Comp., v. 18, 1964, pp. 349-367. MR 29 \#2982.

2. J. H. Bramble \& B. E. Hubbard, "Approximation of solutions of mixed boundary value problems for Poisson's equation by finite differences," J. Assoc. Comput. Mach., v. 12, 1965, pp. 114-123. MR 30 \#1615.

3. V. Thuraisamy, "Approximate solutions for mixed boundary value problems by finitedifference methods," Math. Comp., v. 23, 1969, pp. 373-386.

4. R. S. VARGA, Matrix Iterative Analysis, Prentice-Hall, Englewood, Cliffs, N. J., 1962. MR 28 \#1725. 